\title{
Crystallographic groups with trivial center and outer automorphism group
}

\author{
R. Lutowski, A. Szczepański *
}

\begin{abstract}
Let $\Gamma$ be a crystallographic group of dimension $n$, i.e. a discrete, cocompact subgroup of $\operatorname{Isom}\left(\mathbb{R}^{n}\right)=O(n) \ltimes \mathbb{R}^{n}$. For any $n \geq 2$, we construct a crystallographic group with a trivial center and a trivial outer automorphism group.
\end{abstract}

\section{Introduction}

Let $\Gamma$ be a discrete, cocompact subgroup of $O(n) \ltimes \mathbb{R}^{n}=\operatorname{Isom}\left(\mathbb{R}^{n}\right)$ i.e. a crystallographic group. If $\Gamma$ is a torsion free group, then $M=\mathbb{R}^{n} / \Gamma$ is a flat manifold (that is a compact Riemannian manifold without boundary with the sectional curvature $K_{x}=0$ for any $\left.x \in M\right)$. Moreover $\pi_{1}(M)=\Gamma$.

In $2003 \mathrm{R}$. Waldmüller found a torsion free crystallographic group $\Gamma \subset$ $O(141) \ltimes \mathbb{R}^{141}$ (a flat manifold $M=\mathbb{R}^{141} / \Gamma$ ) with the following properties:

(i) $Z(\Gamma)=\{e\}$,

(ii) $\operatorname{Out}(\Gamma)=\{e\}$,

where $Z(\Gamma)$ is the center of the group $\Gamma$, and $\operatorname{Out}(\Gamma)=\operatorname{Aut}(\Gamma) / \operatorname{Inn}(\Gamma)$ denotes the group of outer automorphisms of $\Gamma$ (see [4, Appendix C] and [5]). Equivalently, $(i)$ means that the abelianization of $\Gamma$ is finite (the first Betti number of $M$ is equal to zero). Moreover, if both conditions $(i)$ and (ii) are satisfied, then the group of affine diffeomorphisms $\operatorname{Aff}(M)$ of the manifold $M$ is trivial (see [2] and [4]).

${ }^{*}$ Both authors are supported by the Polish National Science Center grant 2013/09/B/ST1/04125.

2010 Mathematics Subject Classification: Primary 20H15; Secondary 57S30.

Key words and phrases: Euclidean orbifolds, Crystallographic groups. 
We do not know if there exist such flat manifolds in dimensions less than 141. For example in dimensions up to six such Bieberbach groups do not exist. In this paper we are interested in the existence of not necessarily torsion free crystallographic groups with the above properties. We shall prove that for any $n \geq 2$ there exists a crystallographic group of dimension $n$ which satisfies conditions $(i)$ and $(i i)$.

The main motivation for us is the article [1] of M. Belolipetsky and A. Lubotzky. For any $n \geq 3$ they found an infinite family of hyperbolic compact manifolds of dimension $n$ with the following property: for every manifold $M$ from this family, $\operatorname{Out}\left(\pi_{1}(M)\right)=\{e\}$. Since the center of the fundamental group of a compact hyperbolic manifold is trivial, the above result gives us an infinite family of groups which satisfy conditions $(i)$ and $(i i)$. The construction of the above hyperbolic examples uses the properties of simple Lie groups of $\mathbb{R}$-rank one and, in particular, follows from the existence of non arithmetic lattices.

In our construction the most important are Bieberbach theorems, and specific properties of crystallographic groups.

\section{Crystallographic groups with trivial center and outer automorphism group}

In this part we shall prove our main result. Let $\Gamma$ be a torsion free crystallographic group. From Bieberbach's theorems (see [4, Chapter 2]) we have a short exact sequence of groups

$$
0 \rightarrow \mathbb{Z}^{n} \rightarrow \Gamma \stackrel{p}{\rightarrow} G \rightarrow 0
$$

where $\mathbb{Z}^{n}$ is a maximal abelian subgroup of $\Gamma$ and $G$ is a finite group. Moreover, let $h_{\Gamma}: G \rightarrow \mathrm{GL}(n, \mathbb{Z})$ be the integral holonomy representation defined by the formula

$$
\forall_{g \in G} h_{\Gamma}(g)(e)=\bar{g} e \bar{g}^{-1},
$$

where $\bar{g} \in \Gamma, p(\bar{g})=g$ and $e \in \mathbb{Z}^{n}$. Let

$$
N=N_{\mathrm{GL}(n, \mathbb{Z})}\left(h_{\Gamma}(G)\right)=\left\{X \in \mathrm{GL}(n, \mathbb{Z}) \mid \forall_{f \in h_{\Gamma}(G)} X f X^{-1} \in h_{\Gamma}(G)\right\}
$$


In the case when $Z(\Gamma)=\{e\}$, we have the following commutative diagram ([4, p. 65-69]) with exact rows and columns:

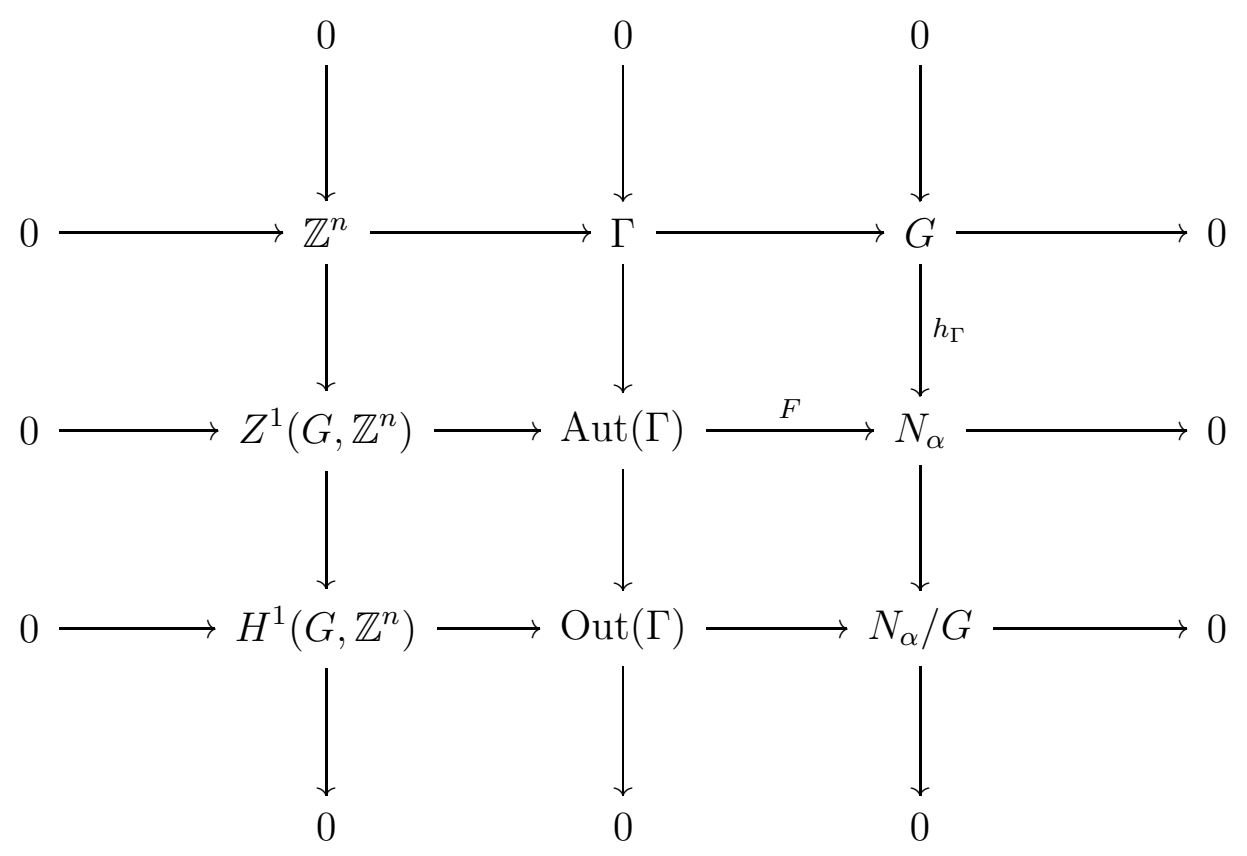

Diagram 1

where $Z^{1}\left(G, \mathbb{Z}^{n}\right)$ is the group of 1 -cocycles. Moreover

$$
N_{\alpha}=\{n \in N \mid n * \alpha=\alpha\}
$$

and $\alpha \in H^{2}\left(G, \mathbb{Z}^{n}\right)$ is the cohomology class of the first row of the diagram. The action $*: N \times H^{2}\left(G, \mathbb{Z}^{n}\right) \rightarrow H^{2}\left(G, \mathbb{Z}^{n}\right)$ is defined by the formula

$$
n *[a]=[n * a],
$$

where $n \in N, a \in Z^{2}\left(G, \mathbb{Z}^{n}\right), \quad[a]$ is the cohomology class of $a$ and

$$
\forall_{g_{1}, g_{2} \in G} n * a\left(g_{1}, g_{2}\right)=n a\left(n^{-1} g_{1} n, n^{-1} g_{2} n\right) .
$$

We have the following proposition.

Proposition 2.1. Aut $(\Gamma)$ is a crystallographic group if and only if $\operatorname{Out}(\Gamma)$ is a finite group. 
Proof: We start with an observation that $Z^{1}\left(G, \mathbb{Z}^{n}\right)$ is a free abelian group of rank $n$ which is a faithful $N_{\alpha}$ module. First, assume that Aut $(\Gamma)$ is a crystallographic group with the maximal abelian subgroup $M$. From [2, Proposition I.4.1], $M$ is the unique normal maximal abelian subgroup of Aut $(\Gamma)$. Hence, $M=Z^{1}\left(G, \mathbb{Z}^{n}\right)$, and $\operatorname{Out}(\Gamma)$ is a finite group. The reverse implication is obvious. This finishes the proof of the proposition.

Let us formulate our main result.

Theorem 2.2. For every $n \geq 2$ there exists a crystallographic group $\Gamma$ of dimension $n$ with $Z(\Gamma)=\operatorname{Out}(\Gamma)=\{e\}$.

\section{Proof:}

We shall need the following lemma.

Lemma 2.3. Let $G, H$ be finite groups and $H \subset G \subset \mathrm{GL}(n, \mathbb{Z})$. If the group $N_{\mathrm{GL}(n, \mathbb{Z})}(H)$ is finite, then $N_{\mathrm{GL}(n, \mathbb{Z})}(G)$ is finite.

Proof of Lemma: From the assumption, $\operatorname{Aut}(H)$ and $\operatorname{Aut}(G)$ are finite. Moreover, we have monomorphisms:

$$
N_{\mathrm{GL}(n, \mathbb{Z})}(H) / C_{\mathrm{GL}(n, \mathbb{Z})}(H) \stackrel{\bar{\phi}}{\rightarrow} \operatorname{Aut}(H)
$$

and

$$
N_{\mathrm{GL}(n, \mathbb{Z})}(G) / C_{\mathrm{GL}(n, \mathbb{Z})}(G) \stackrel{\bar{\phi}}{\rightarrow} \operatorname{Aut}(G),
$$

where $\bar{\phi}$ is induced by $\phi(s)(g)=s g s^{-1}, g \in G, s \in \mathrm{GL}(n, \mathbb{Z})$. Since $C_{\mathrm{GL}(n, \mathbb{Z})}(G) \subset$ $C_{\mathrm{GL}(n, \mathbb{Z})}(H)$, our Lemma is proved.

Corollary 2.4. If $|\operatorname{Out}(\Gamma)|<\infty$, then $|\operatorname{Out}(\operatorname{Aut}(\Gamma))|<\infty$.

Lemma 2.5. Assume $Z(\Gamma)=\{e\}$, then

1. $H^{1}\left(G, \mathbb{Z}^{n}\right) \simeq\left(\mathbb{Q}^{n} / \mathbb{Z}^{n}\right)^{G}=H^{0}\left(G, \mathbb{Q}^{n} / \mathbb{Z}^{n}\right)$

2. $Z^{1}\left(G, \mathbb{Z}^{n}\right) \simeq\left\{m \in \mathbb{Q}^{n} \mid \forall_{g \in G}\right.$ gm $\left.-m \in \mathbb{Z}^{n}\right\}=A^{0}(\Gamma)$ as $N_{\alpha}$ modules;

3. $A(\Gamma)=N_{\mathrm{Aff}\left(\mathbb{R}^{n}\right)}(\Gamma)=\left\{a \in \operatorname{Aff}\left(\mathbb{R}^{n}\right) \mid \forall_{\gamma \in \Gamma} a \gamma a^{-1} \in \Gamma\right\} \simeq \operatorname{Aut}(\Gamma)$. 
We have the following modification of the Diagram 2.

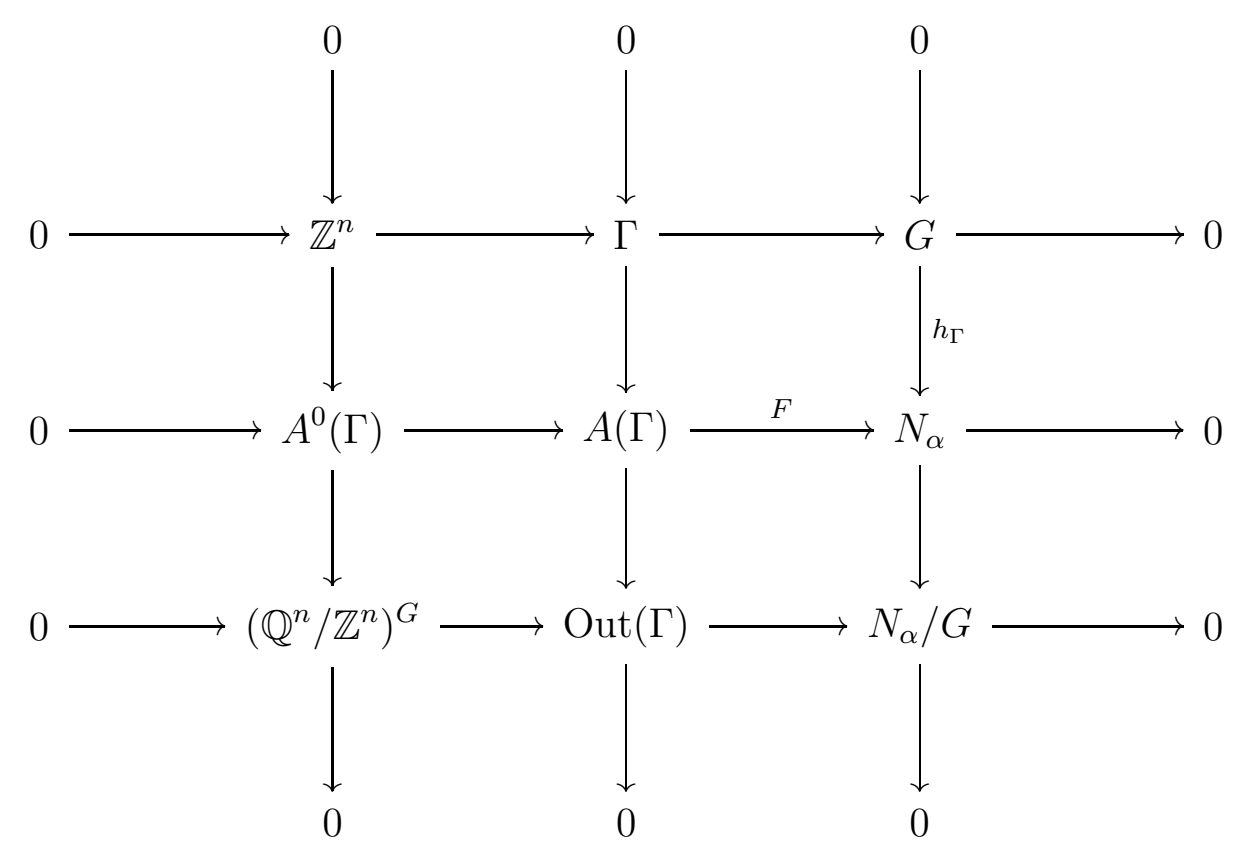

Diagram 2

Let $\Gamma$ be a crystallographic group of rank $n$ with trivial center and holonomy group $G$. Moreover, assume that the group $H^{1}\left(G, \mathbb{Z}^{n}\right)=\{e\}$, and the group $\operatorname{Out}(\Gamma)$ is finite. Inductively, put $\Gamma_{0}=\Gamma$ and $\Gamma_{i+1}=\mathrm{A}\left(\Gamma_{i}\right)$, for $i \geq 0$.

Lemma 2.6. $\exists N$ such that $\Gamma_{N+1}=\Gamma_{N}$.

Proof: We start from observations that for $i>0, \Gamma_{i}$ is a crystallographic group, $Z\left(\Gamma_{i}\right)=\{e\}$ and $M_{0}=M_{i}$, where $M_{i}=A^{0}\left(\Gamma_{i-1}\right) \subset \Gamma_{i}$ is the maximal abelian normal subgroup (a subgroup of translations). Let $G_{i}=\Gamma_{i} / M_{i}$. From definition we can consider $\left(G_{i}\right)$ as a nondecreasing sequence of finite subgroups of $\operatorname{GL}(n, \mathbb{Z})$. From Bieberbach theorems [4, Chapter 2] and from Diagrams 1 and 2, there is only a finite number of possibilities for $G_{i}$. Hence $\exists N \in \mathbb{N}$ such that $\forall_{i>N} G_{i}=G_{N}$. This finishes the proof.

Example 2.1. Let $\Gamma_{1}=G_{1} \ltimes \mathbb{Z}^{2}$ be the crystallographic group of dimension 2 with holonomy group $G_{1}=D_{12}$, where

$$
D_{12}=\operatorname{gen}\left\{\left[\begin{array}{cc}
0 & -1 \\
1 & -1
\end{array}\right],\left[\begin{array}{cc}
-1 & 0 \\
0 & -1
\end{array}\right],\left[\begin{array}{ll}
0 & 1 \\
1 & 0
\end{array}\right]\right\}
$$


is the dihedral group of order 12 . Moreover, let $\Gamma_{2}=G_{2} \ltimes \mathbb{Z}^{3}$ be the crystallographic group of dimension 3 , with holonomy group $G_{2}=S_{4} \times \mathbb{Z}_{2}$ generated by matrices

$$
\left[\begin{array}{ccc}
0 & 1 & 0 \\
0 & -1 & -1 \\
1 & 1 & 0
\end{array}\right], \quad\left[\begin{array}{ccc}
0 & 0 & 1 \\
0 & -1 & -1 \\
-1 & 0 & 1
\end{array}\right]
$$

Here $S_{4}$ denotes the symmetric group on four letters.

For $i=1,2$ we have

$$
N_{\mathrm{GL}\left(n_{i}, \mathbb{Z}\right)}\left(G_{i}\right)=G_{i}
$$

and

$$
H^{1}\left(G_{i}, \mathbb{Z}^{n_{i}}\right)=0
$$

where $n_{i}$ is the rank of $\Gamma_{i}$. Hence $A\left(\Gamma_{i}\right)=\Gamma_{i}$, and $\operatorname{Out}\left(\Gamma_{i}\right)=\{e\}$, for $i=1,2$.

Now we are ready to finish the proof of Theorem 2.2. The cases $n=2,3$ are done in the above example. Assume $n \geq 4$. Let $n=2 k+3 i$, where $i \in\{0,1\}$. Put $\Gamma^{\prime}=\Gamma_{1}^{k} \times \Gamma_{2}^{i}$. Then $\Gamma^{\prime}$ is centerless and by [3, Theorem 3.4] the bottom exact sequence of the Diagram 2 looks as follows

$$
0 \rightarrow 0 \rightarrow \operatorname{Out}\left(\Gamma^{\prime}\right) \rightarrow S_{k} \rightarrow 0
$$

Hence, $\Gamma^{\prime}$ satisfies the assumption of Lemma 2.6 and the sequence $\Gamma_{0}=$ $\Gamma^{\prime}, \Gamma_{i+1}=\mathrm{A}\left(\Gamma_{i}\right)$ stabilizes, i.e., $\exists N$ such that $\forall_{i \geq N} \quad \Gamma_{i}=\Gamma_{N}$. Moreover, $\operatorname{Out}\left(\Gamma_{N}\right)=\{e\}$ and $Z\left(\Gamma_{N}\right)=\{e\}$.

\section{References}

[1] M. Belolipetsky, A. Lubotzky, Finite groups and hyperbolic manifolds, Invent. Math. 162, (2005) no. 3459 - 472

[2] L. S. Charlap, Bieberbach Groups and Flat Manifolds, Universitext, Springer-Verlag, New York, 1986

[3] R. Lutowski, On symmetry of flat manifolds, Exp. Math. 18 (2009), no. $3,201-204$

[4] A. Szczepański, Geometry of the crystallographic groups, Algebra and Discrete Mathematics Vol. 4, World Scientific, Shanghai 2012 
[5] R. Waldmüller, A flat manifold with no symmetries, Experiment. Math. 12 (2003), no. $1,71-77$

Institute of Mathematics

University of Gdańsk

ul. Wita Stwosza 57

80-952 Gdańsk

Poland

E-mail: rlutowsk@mat.ug.edu.pl, matas@univ.gda.pl 